Revista de Psicología de la PUCP. Vol. XVIII, 1, 2000.

\title{
Evaluación del bienestar psicológico en estudiantes adolescentes argentinos ${ }^{1}$
}

\author{
María Martina Casullo ${ }^{2}$ \\ Alejandro Castro Solano ${ }^{3}$ \\ Universidad de Buenos Aires
}

\begin{abstract}
El objetivo de este estudio es el desarrollo de una escala breve para la evaluación del bienestar psicológico en adolescentes, la revisión de sus características psicométricas. En el mismo sentido se intentó verificar la presencia de diferencias individuales entre el sexo, la edad y el lugar de residencia de los sujetos evaluados. Los participantes son adolescentes entre las edades de 13 a 18 años ( $\mathrm{N}=1270)$ de tres regiones de la Argentina (Región Metropolitana, Noroeste y Patagonia). Los instrumentos administrados son la escala de bienestar (BIEPS-J), otras escalas tradicionales para la evaluación de la satisfacción (Escala SWLS y D-T) y un listado de síntomas psicopatológicos (SCL-90). Los resultados señalan que las escalas clásicas que evalúan satisfacción con la vida resultan indicadores poco válidos para una evaluación del bienestar psicológico autopercibido, considerando las dimensiones teóricas comentadas. Las variables género, edad y contexto sociocultural no parecen afectar la percepción subjetiva del bienestar psicológico.

Palabras clave: Bienestar psicológico, satisfacción con la vida, evaluación psicológica, adolescencia.
\end{abstract}

Psychological assessment of psychological well being in Argentine adolescent students

The main goal of this study is to develop a brief scale to assess perceived well being in adolescent population. Besides verifying psychometric properties, we identified individual differences between genders, context and age. Participants were adolescent students recruited in three different areas of Argentina (Metropolitan area -Buenos Aires-, Norwest area - Tucuman - and Southwest area Patagonia-). aged 13 Th. 18. Instruments administered consisted of BIEPS (well being scale) other classics instruments to assess life satisfaction (SWLS and D-T) and a symptom check list (SLC90). Results show that classic instruments to assess well being resulted in less reliable instruments to assess perceived life satisfaction considering other theories. Gender, age and context don't seem to affect subjective perception of psychological well being.

Key Words: Well being, life satisfaction, psychological assessment, adolescence.

1

Se agradece la participación de los psicólogos Norma Contini de González ( Tucumán) y Flavio D'Angelo (Cipolletti, Río Negro).

Doctora en Psicología. Directora de los proyectos de investigación UBACyT 007 y CONICET PIP 4102. Instituto de Investigaciones. Miembro de la carrera del investigador de CONICET. Profesora regular de la Facultad de Psicología UBA. Independencia 3065. 1225 Buenos Aires. Argentina. Correo electrónico: casullo@psi.uba.ar.

Doctor en Psicología. Integrante deI equipo de investigación de CONICET. Correo electrónico: alex1@cvtci.com.ar. 

¿Por qué algunas personas se sienten mejor que otras? ¿Por qué algunos están más satisfechos con la vida? ¿Cuáles son las causas de la insatisfacción? Estas preguntas han servido de guía de los estudios actuales sobre la satisfacción vital y el bienestar psicológico. Durante años el tema fue objeto de debate filosófico y sociológico; se creyó que el sentirse satisfecho con la vida estaba muy relacionado con la inteligencia, la apariencia física o las buenas condiciones económicas. Hoy en día se sabe que el mayor o menor bienestar subjetivo no está tan relacionado con esas variables, sino con la subjetividad de los individuos (Diener, Suh y Oishi, 1997).

¿Qué entendemos por bienestar? Una persona tiene alto bienestar si experimenta satisfacción con su vida, si frecuentemente su estado anímico es bueno y sólo ocasionalmente experimenta emociones poco placenteras como tristeza o rabia. La literatura de investigación señala que la satisfacción es un constructo triárquico. Por un lado tenemos los estados emocionales (afecto positivo y afecto negativo) y por el otro el componente cognitivo. Los estados emocionales son más lábiles y momentáneos, al mismo tiempo son independientes. El tener una dosis alta de afectividad positiva no implica tener una dosis alta de afectividad negativa. El componente cognitivo de la satisfacción se denomina bienestar y es resultado de la integración cognitiva que las personas realizan acerca de cómo les fue (o les esta yendo) en el transcurso de su vida.

Las definiciones del bienestar planteadas por los diferentes investigadores en sus estudios no resultan del todo explícitas. En términos generales están caracterizadas por el tipo de instrumento utilizado para su medición (Diener, 1994). Veenhoven (1991) define al bienestar como 
el grado en que un individuo juzga su vida "como un todo" en términos favorables. Andrews y Withey (1976) señalan que el bienestar subjetivo tomando en cuenta los aspectos cognitivos junto con los afectivos (afecto positivo y negativo). Campbell, Converse y Rodgers (1976) definen a la satisfacción como una dimensión cognitiva, resultante de la discrepancia entre las aspiraciones y los logros alcanzados. Para estos autores la satisfacción implica un juicio cognitivo, mientras que la felicidad es una experiencia de afecto positivo y/o negativo. Diener (1994) sostiene que el término felicidad es evitado en la investigación científico-académica porque alude a su significación popular, es decir, la experiencia de bienestar, de afecto positivo y negativo en su dimensión diacrónica. El autor comenta que el bienestar subjetivo posee componentes estables y cambiantes al mismo tiempo. La apreciación de los eventos se modifica en función del afecto negativo y positivo predominante. El bienestar tiene estabilidad en el largo plazo, a pesar de experimentar variaciones circunstanciales en función de los afectos predominantes. Para Diener et al. (1997) el bienestar es entendido como el grado en que una persona evalúa su vida, incluyendo como componentes la satisfacción con la vida, con el matrimonio, la ausencia de depresión y la experiencia de emociones positivas. El componente cognitivo, a los efectos de la investigación científica, parece más relevante, dado que su principal característica es ser estable, se mantiene en el tiempo e impregna la vida global de los sujetos. Por otro lado, el componente afectivo es mucho más lábil, momentáneo y cambiante. Los investigadores en el campo de la satisfacción se han volcado generalmente al estudio del componente estable de la satisfacción.

¿Por qué es importante el estudio del bienestar? En primer lugar es de interés para la ciencia psicológica poder determinar por qué algunas personas se sienten muy satisfechas con la vida mientras que otras no. ¿Por qué la gente está más o menos satisfecha?, ¿Qué condiciones objetivas hacen que esa satisfacción se eleve, disminuya o se mantenga? y ¿cómo mejora la calidad de vida de las personas? ¿Puede elevarse o mejorarse la satisfacción? (Strack y Argyle, 1991). 
En un nivel psicológico individual podemos apreciar que la psicología se ha ocupado, tradicionalmente, más de aspectos patológicos, enfermos y desajustados, de todo aquello que tenga que ver con el malestar (Veenhoven, 1988, 1991). A lo largo de años de investigación se descuidó el efecto que el bienestar y la felicidad tenían sobre el funcionamiento psicológico personal. Los desarrollos de la psicología en el terreno de la salud mental elaboraron concepciones que explicaban el malestar de las personas, descuidando los aspectos salugénicos (Ryff y Keyes, 1995).

Diferentes investigaciones han resaltado que aquellos individuos más felices y satisfechos sufren menos malestar, tienen mejores apreciaciones personales, un mejor dominio del entorno y poseen mejores habilidades sociales para vincularse con las demás personas. Podríamos caracterizar la satisfacción y la felicidad como indicadores de un buen funcionamiento mental (Argyle, 1987). Taylor y Brown (1988) enfatizan la importancia de las "ilusiones positivas" como criterio de salud mental, es decir, considerar que el presente y el futuro pueden ser mejores, sin importar las condiciones objetivas que impone la realidad. Veenhoven (1995) al revisar la evidencia que los individuos felices superan en una proporción de 3 a 1 a los que no lo son, llegó incluso a caracterizar a la felicidad y al bienestar psicológico como disposiciones bio-psicológicas naturales. Así como el estado natural biológico del organismo es la salud, en el área psicológica esa disposición la constituye el bienestar subjetivo.

\section{Algunos hallazgos de las investigaciones sobre bienestar}

Se ha constatado que las variables sociodemográficas no son buenos predictores del bienestar. En las últimas dos décadas los investigadores se han volcado al estudio de variables personológicas. Los teóricos de la personalidad como Costa y McCrae (1980) han encontrado que las dimensiones de extroversión están relacionadas con el afecto positivo y por ende con la satisfacción y que la dimensión neuroticismo se relaciona con el afecto negativo y la insatisfacción. Estos autores interpretan que las personas mantienen un nivel estable de satisfacción a lo largo de sus 
vidas, resultado de la interacción de las dimensiones de personalidad latentes. Los trabajos de investigación que siguen esta metodología tienden a replicar los resultados originales encontrados por Costa y McCrae (1980) ya sea mediante estudios correlacionales o experimentales (Larsen y Ketelaar, 1991). En general es aceptado que las dimensiones del afecto positivo y negativo son independientes y responden a las disposiciones de Neuroticismo y Extroversión (Costa y McCrae, 1984; Hotard, McFeather, McWhirter y Stegall, 1989). Aunque algunos autores afirman que las correlaciones establecidas entre los rasgos y la satisfacción son fuertes, todavía no existe evidencia del nexo causal o unidireccionalidad entre ambas variables (Emmons y Diener, 1985; Headey y Wearing, 1989). Investigadores europeos como Eysenck y Gray afirman que las diferencias de comportamiento entre los extravertidos e introvertidos se deben a las estructuras neurológicas (Avia y Vázquez, 1998). Los extravertidos son más sensibles a las señales de recompensa y los neuróticos más sensibles al castigo. Las investigaciones experimentales de Larsen y Ketelaar (1991) probaron lo fácil que es producir estados de ánimo negativo en los más neuróticos y estados de ánimo positivo en los más extrovertidos; parecería, dicen los autores, que los neuróticos estuvieran preparados para reaccionar con fuertes emociones desagradables y los extrovertidos con un intenso afecto positivo. Por otro lado, los hallazgos de investigaciones realizadas con gemelos separados al momento del nacimiento indican que tanto el afecto positivo como negativo tienen un fuerte componente temperamental. Los investigadores demostraron que alrededor del $50 \%$ de la variación entre individuos era atribuible a diferencias temperamentales, es decir, hereditarias y el resto se debía a componentes ambientales (Lykken y Tellegen, 1996; Tellegen et al., 1988).

Costa, McCrae y Zonderman (1987) en un estudio longitudinal verificaron que aquellas personas que cambiaban de estado civil, residencia, empleo, etc, comparadas con las que mantenían estas condiciones estables no mostraban cambios importantes en su bienestar. Estos eventos podían alterar momentáneamente su nivel de bienestar, pero al poco tiempo volvían a la línea de base. Este argumento sugiere 
Evaluación del bienestar psicológico en estudiantes adolescentes argentinos

que el bienestar parece estar relacionado con los componentes temperamentales de las personas.

Veenhoven (1993), desde el campo de la sociología, sostiene que la satisfacción es un constructo estable en el corto plazo pero no en el largo plazo, al menos de modo inmutable como aseveran los investigadores de los rasgos. Si bien es cierto que la satisfacción responde a un proceso de reevaluación permanente, también es cierto que las personas no actualizan su juicio a menos que estén realmente presionadas para hacerlo. En conclusión, el autor cita una cantidad suficiente de datos empíricos concluyendo que la satisfacción no es una disposición fija e inmutable.

- La satisfacción no es temporalmente estable. Los individuos evalúan continuamente su situación personal, tanto a nivel individual como social.

- No es situacionalmente consistente. Las personas no son igual de felices en mejores o en peores condiciones. La felicidad promedio es superior en los países que tienen mejores condiciones de vida.

- La felicidad no es una vivencia netamente interna e individual. Si bien es cierto que existen ciertas disposiciones individuales, existen efectos ambientales que interactúan con las variables personales.

Desde la perspectiva de la psicología cultural al analizar el bienestar que experimentan las personas en diferentes contextos culturales, los estudios revelan que no se observan diferencias significativas en los niveles de satisfacción con la vida en aquellos países que tienen más derechos civiles, riqueza y libertades individuales, comparados con los menos desarrollados y más pobres (Diener y Diener, 1995). Se verifican grandes diferencias en los niveles de bienestar si comparamos países muy pobres cuyas condiciones de vida mínima no han sido satisfactoriamente alcanzadas y los países más desarrollados. Los estudios consultados revelan que una vez satisfechas las necesidades básicas de las personas, no existen grandes diferencias en los niveles de satisfacción con la vida entre las diferentes naciones (Avia y Vázquez, 1998; Veenhoven, 1991). Cummins (1998) realizó un metanálisis de los artículos 
publicados que reseñaban información sobre las diferencias en el nivel de satisfacción entre los países. Los resultados de su estudio señalaron que más de las tres cuartas partes de la población mundial se encontraba satisfecha con sus vidas.

Si tomamos en cuenta la diferencia entre países individualistas y colectivistas siguiendo la línea de trabajo propuesta por Triandis (1989, 1994), podemos ver que en aquellos países más individualistas, tanto la autoestima como el optimismo están muy relacionados con el bienestar (Lucas, Diener y Suh, 1996). En otro estudio, comparando diferentes países, Diener y Diener (1995) encontraron que el individualismo de las naciones correlacionaba positivamente con la satisfacción con el grupo de amigos, siendo este predictor menos importante en países más colectivistas. Los autores también señalan que contrariamente a las hipótesis previas, el colectivismo de las naciones no estaba asociado con el grado de satisfacción con la familia. La conclusión del estudio comentado indica que los participantes de más de 30 países, estaban en términos generales muy satisfechos con sus vidas, con ellos mismos y con las áreas vitales familia y amigos. Sin embargo Diener et. al. (1997) comentan que muchas investigaciones transculturales no han sido debidamente replicadas. Los autores aseguran que todavía no sabemos demasiado acerca de las relaciones entre la personalidad y el bienestar, si este último es producto de determinantes ambientales, atribuibles al contexto o si está relacionado con componentes biológicos temperamentales.

¿Qué contexto da lugar a una mejor calidad de vida? ¿Las grandes ciudades o las pequeñas aldeas rurales? Desde la sociología se ha caracterizado los factores que inciden sobre la calidad de vida y el bienestar en las ciudades: alta densidad poblacional, aislamiento y desorganización social como resultado de la vida en la gran urbe (Adams, 1992). Las consecuencias psicológicas de estos factores resultan claras: los ámbitos más urbanizados traen consecuencias negativas para las relaciones sociales y para la salud mental (Sarason, 1974). El modelo sistémico sostiene que al ser los ámbitos más estables (tal el caso de las 
pequeñas ciudades), la población registra menores cambios, se incrementan las redes sociales y por lo tanto la satisfacción y la calidad de vida aumenta (Fischer, 1973). Por otro lado, el modelo de desarrollo lineal predice que en tanto el tamaño y la diversidad de una población aumenta, las personas se vuelven mas individualistas, egoístas y alienadas de los demás, por lo tanto la satisfacción con la vida para esa población disminuye, experimentando las personas sentimientos de extrañamiento, aislamiento y ausencia de control sobre sus vidas (Milgram, 1970).

Existen muchas opiniones, teorías y modelos, pero pocas contrastaciones empíricas. Se han localizado muy pocos estudios que hayan hecho una verificación empírica de estas cuestiones. Encontramos estudios con resultados incongruentes. Dohrenwend y Dohrenwend (1974) verificaron una alta prevalencia de trastornos físicos y psiquiátricos en las áreas más urbanas. Los estudios de Mueller (1981) y Neff (1983) señalan las mismas conclusiones. Hynson (1975) verificó en una muestra nacional de adultos mayores de 60 años, que los residentes de ámbitos rurales estaban más satisfechos con sus vidas que los que vivían en las grandes ciudades. Srole (1972, 1978), por el contrario, encontró más desórdenes mentales en las áreas rurales.

Para Adams (1992) la cuestión es mucho más difícil que establecer si los ámbitos urbanos son más o menos promotores de bienestar que los rurales, ya que intervienen variables moderadoras diversas. $\mathrm{El}$ autor no encontró grandes diferencias en los niveles de satisfacción percibida en ambos contextos. La variable que más diferenciaba ambos entornos era la densidad poblacional, a mayor cantidad de personas extrañas en el vecindario próximo decrece la expectativa de control sobre el medio y por lo tanto existe un menor nivel de bienestar. En la misma línea están los hallazgos de Sauer, Shenan, y Boymel (1976) quienes encontraron que no existía relación entre lugar de residencia y satisfacción. Kushman y Lane (1980) no hallaron diferencias significativas entre residentes de ámbitos urbanos y rurales en una muestra de adultos del estado de California. Crider, Willits y Kanagy (1991) encontraron poco apoyo empírico a la tesis idílica de los habitantes del campo; satisfacción y lugar de 
residencia parece que no están relacionados. Los estudios de Beiser y Collomb (1981), Cheng (1989) y Harris (1984) también abogan por la tesis de la no diferencia entre los niveles de bienestar entre gente que vive en hábitats urbanos y rurales.

Pagliccia, Apland y Kazanjian (1995) sostienen que el impacto del tamaño de las ciudades debe analizarse desde la experiencia de urbanización percibida por las propias personas, más importante que los indicadores objetivos.

\section{Una concepción alternativa del bienestar psicológico}

La mayoría de las investigaciones sobre bienestar psicológico no han tenido un marco teórico claro como respaldo. En este campo existe una cantidad de datos empíricos, pero muy pocos modelos teóricos que guíen la construcción de los instrumentos y la interpretación de los resultados de las investigaciones. Los investigadores consideran que el bienestar se operacionaliza fácilmente a través de los índices de afecto positivo o negativo o las escalas unidimensionales de satisfacción, ignorando la adjudicación de significado de los actos humanos, entendido como el sentido de orden o coherencia en la existencia personal. Algunos autores proponen que el oficio de vivir, en un determinado momento, supone desarrollar un sentido de integridad, una apreciación de por qué y cómo uno ha vivido (Reker, Peacock y Wong, 1987). En esta línea se ubican los trabajos de Pychyl y Little (1998) quienes estudian los constructos relacionados con las metas personales y proponen que los proyectos tienen dos funciones: una instrumental relacionada con la eficacia y el alcance de la felicidad y otra más simbólica, relacionada con la consistencia y que da por resultado el significado asignado al proyecto de vida.

En la misma línea, Ryff (1989) critica los estudios clásicos sobre satisfacción; expresa que en general se ha considerado el bienestar psicológico como la ausencia de malestar o de trastornos psicológicos, ignorando las teorías sobre la autorrealización, el ciclo vital, el 
funcionamiento mental óptimo y el significado vital. La autora plantea serias dudas sobre la unidimensionalidad del constructo bienestar psicológico, señalando su multidimensionalidad (Ryff y Keyes, 1995). Clásicamente, el bienestar psicológico fue identificado con la emocionalidad positiva y la ausencia de emociones negativas. Una persona se siente feliz o satisfecha con la vida si experimenta durante más tiempo y en mayor frecuencia mayor cantidad de afecto positivo. Implícitamente el bienestar está identificado con los afectos o la personalidad (Schumutte y Ryff, 1997). Los rasgos de personalidad básicos hacen alusión a la emocionalidad positiva como característica estable de la personalidad, de allí que no resulte extraño que el bienestar esté correlacionado negativamente con el neuroticismo, según aseveran los teóricos de los cinco factores.

El estudio del bienestar debe tomar en cuenta la autoaceptación de si mismo, un sentido de propósitos o significado vital, el sentido de crecimiento personal o compromiso y el establecimiento de buenos vínculos personales. El bienestar es un constructo mucho más amplio que la simple estabilidad de los afectos positivos a lo largo del tiempo, denominado por la sabiduría popular como felicidad (Schumutte y Ryff, 1997).

El bienestar psicológico es una dimensión fundamentalmente evaluativa que tiene que ver con la valoración del resultado logrado con una determinada forma de haber vivido. Ryff ofrece una interesante perspectiva uniendo las teorías del desarrollo humano óptimo, el funcionamiento mental positivo y las teorías del ciclo vital. Para la autora el bienestar tiene variaciones importantes según la edad, el sexo y la cultura; pudo verificar en diferentes estudios que el bienestar psicológico está compuesto por seis dimensiones bien diferenciadas: (a) una apreciación positiva de si mismo; (b) la capacidad para manejar de forma efectiva el medio y la propia vida; (c) la alta calidad de los vínculos personales; (d) la creencia de que la vida tiene propósito y significado; (e) el sentimiento de que se va creciendo y desarrollándose a lo largo de la vida y (f) el sentido de autodeterminación. Se pudo verificar mediante 
técnicas de análisis factorial confirmatorio, en población general norteamericana, la existencia de estas seis dimensiones del bienestar (Ryff y Keyes, 1995).

En la misma línea de orientación salugénica del estudio del funcionamiento mental óptimo, se encuentran los trabajos de Antonovsky sobre el sentido de coherencia (SOC, Antonovsky, 1988). El SOC es definido como una orientación global que expresa que una persona tiene recursos suficientes disponibles como para afrontar las demandas del medio, puede percibir los desafíos que lo movilizan y lo orientan positivamente hacia la vida y puede comprender la información del medio de manera coherente y clara y no se ve invadida por la alta carga de estímulos. Una persona con alto sentido de coherencia experimenta menos emociones negativas tales como depresión y ansiedad y puede hacer frente a los estímulos estresantes de forma más acabada. En el mismo sentido esta orientación global de la personalidad es aquello que se intenta desarrollar en una psicoterapia, ya que el bienestar producto del tratamiento muchas veces consiste en otorgar mayor significado a la vida en función del principio de realidad, es decir, lograr que la persona desarrolle cierto sentido de coherencia. Frenz, Carey y Jorgensen (1993) comentan que a pesar de que el constructo resulta interesante, ha sido bastante criticado; varios autores coinciden en considerarlo la cara opuesta del neuroticismo, de allí que muchos estudios han verificado importantes relaciones con la emocionalidad negativa y positiva.

\section{Objetivos de la investigación}

Este estudio tiene como objetivos principales, desarrollar un instrumento para la medición del bienestar psicológico sobre la base de las propuestas teóricas de Ryff, teniendo en consideración los aspectos salugénicos que, generalmente, no han sido tomados en cuenta en estudios previos sobre el tema; y determinar la validez y confiabilidad de la escala para realizar evaluaciones psicológicas del bienestar en adolescentes argentinos que viven en diferentes contextos culturales. 


\section{Metodología}

Sobre la base de cinco de las seis dimensiones propuestas por Ryff se redactaron 36 ítemes intentando operacionalizar cada una de ellas. Esa versión original fue entregada a tres psicólogos y a una muestra piloto integrada por 20 adolescentes, con la finalidad de que determinaran su grado de acuerdo respecto a la validez de contenido de los reactivos. La versión final a emplear quedó compuesta por los ítemes en los que se encontró un $75 \%$ de acuerdo entre los jueces consultados (autonomía, control, vínculos, proyectos, aceptación de sí mismo).

En segundo lugar analizamos las características psicométricas de la escala y luego presentamos un estudio descriptivo-correlacional que intenta verificar que las dimensiones del bienestar psicológico consideradas (control, vínculos, proyecto y aceptación de sí) permiten diferenciar a jóvenes sanos respecto de otros que desarrollaron trastornos psicológicos.

\section{Participantes}

Participaron del estudio 1270 adolescentes, varones y mujeres, entre 13 y 18 años. Los mismos asistían a institutos de educación secundaria en tres diferentes áreas geográficas de la República Argentina. El grupo original estaba compuesto por 1320 participantes. Algunos fueron eliminados por no haber completado la totalidad de la batería y otros por no responder a los controles de validez de las técnicas utilizadas. La muestra fue intencional ya que participaron del estudio aquellas instituciones que tenían convenio de cooperación con la Universidad de Buenos Aires.

Los participantes fueron agrupados de la siguiente manera:

Grupo 1 - Región Metropolitana (Ciudad de Buenos Aires). Pertenecían a este grupo un total de 498 participantes que asistían a escuelas públicas . La ciudad de Buenos Aires es el área metropolitana 
de la República Argentina, es la región de mayor densidad poblacional y constituye el centro comercial y urbano más importante del país. El 53\% eran mujeres y el $47 \%$ eran varones . La media de edad para los varones era de $15.98(D E=1.62)$ y para las mujeres de $15.82(D E=1.54)$. La mayor proporción de participantes, tanto para varones como para mujeres, se encontraba entre los 16 y los 18 años (62\%), existiendo un menor porcentaje de estudiantes que tenían entre 13 y 15 (38\%).

Grupo 2 - Región Noroeste (Tucumán). Pertenecen a este grupo un total de 453 participantes que asistían escuelas públicas en la Ciudad de Tucumán. La ciudad de Tucumán pertenece a la región denominada del Noroeste Argentino. Está ubicada a mas de 1.100 kilómetros de distancia de la capital. Se caracteriza por tener un menor grado de urbanización que la ciudad de Buenos Aires, no obstante, nuclea las actividades comerciales de la región ya que es capital de provincia. Es la provincia más pequeña de todo el territorio nacional y la mayor parte del territorio es región de alta montaña y no se encuentra habitado. El $55 \%$ eran mujeres y el $45 \%$ eran varones. La media de edad para los varones era de $15.09(D E=1.44)$ y para las mujeres de $14.71(D E=1.51)$. La mayor proporción de participantes, tanto para varones como para mujeres, se encontraba entre los 13 y 15 años (63\%), existiendo un menor porcentaje de estudiantes que tenían entre 16 y 18 años (37\%).

Grupo 3 - Región Patagonia (Alto Valle, ciudades de Cipolletti y Neuquén). Pertenecen a este grupo un total de 319 estudiantes adolescentes que concurren a escuelas medias en las ciudades de Neuquén y Cipolletti, al sur del país. Pertenece a la Región denominada del Alto Valle de Rio Negro. Componen la muestra un $59 \%$ de adolescentes mujeres y un 41 $\%$ de varones. La media de edad para los varones era de 15.04 ( $D E=$ 1.38) y para las mujeres de $15.20(D E=1.45)$. La mayor proporción de participantes, tanto para varones como para mujeres, se encontraba entre los 13 y 15 años (60\%), existiendo un menor porcentaje de estudiantes que tenían entre 16 y 18 años (40\%). 
Evaluación del bienestar psicológico en estudiantes adolescentes argentinos

\section{Instrumentos}

\section{Escala de Bienestar Psicológico (BIEPS)}

Se diseñó un instrumento ad-hoc para la evaluación del bienestar psicológico en los jóvenes, según los lineamientos teóricos presentados, integrado por 20 ítemes. Se consideraron las dimensiones autonomia (capacidad de actuar de modo independiente), control de situaciones (sensacion de control y autocompetencia), vínculos psicosociales (calidad de las relaciones personales), proyectos (metas y propositos en la vida) y aceptacion de si (sentimiento de bienestar con uno mismo). Se emplearon cuatro ítemes para cada una de las escalas de modo que el contenido de cada uno de ellas refleje cada una de las dimensiones del bienestar consideradas. Cada frase podía ser respondida en un formato de respuesta likert en tres opciones de respuesta. Se obtuvo una puntuación total y una para cada subescala.

Para verificar la pureza psicométrica de cada uno de los ítemes se llevó a cabo un análisis factorial de componentes principales, con rotación varimax de sus elementos. El proceso fue llevado a cabo de forma iterativa eliminándose en cada paso los ítemes que tuvieran peso similar en más de un factor. La solución factorial final constaba de cuatro factores que explicaban más del $50 \%$ de la varianza de las puntuaciones. Los factores resultantes fueron control de situaciones, vínculos, proyectos y aceptación de sí. El factor autonomía no se pudo verificar en la solución factorial, resultando muy confuso, por lo tanto fue eliminado. La confiabilidad total de la escala fue aceptable alcanzando valores de 0.74 . Sin embargo, estos valores descendían sensiblemente si se consideraba cada una de las escalas por separado dado que cada subescala estaba compuesta por muy pocos elementos (alrededor de 0.50). En la sección de resultados se presenta la matriz factorial obtenida y los estudios correlacionales con otros instrumentos de evaluación psicológica que verifican la validez de la misma. 


\section{Listado de síntomas (Symptom Checklist - 90 - R)}

Es un listado de 90 síntomas clínicos, agrupados en nueve áreas sintomáticas; es autoadministrable. El encuestado tenía que responder si le había molestado o preocupado el síntoma listado durante la última semana, en una escala tipo likert con cinco opciones de respuesta (desde nada hasta mucho). Las dimensiones fueron las siguientes:

1. Somatización (quejas o molestias relacionadas con el mal funcionamiento del cuerpo. Dolor y disconformidad).

2. Obsesiones y Compulsiones (identifica pensamientos, impulsos y acciones intrusivas que son experimentadas como egodistónicas y displacenteras).

3. Sensibilidad Interpersonal (sentimientos de inferioridad e inadecuación, particularmente en las relaciones personales).

4. Depresión (síntomas de estado de ánimo disfórico, aislamiento, falta de motivación, desesperanza y pérdida de la energía).

5. Ansiedad. (signos generales de la ansiedad. Ataques de pánico, temor, nerviosismo y correlatos somáticos de la ansiedad).

6. Hostilidad (sentimientos, afectos y acciones relacionadas con la ira y agresión).

7. Ansiedad Fóbica (respuesta de miedo y ansiedad desproporcionada e irracional al estímulo. El sujeto toma conductas de carácter evitativo).

8. Ideación Paranoide (conductas paranoides; estilo de pensamiento desordenado y confuso. Hostilidad, suspicacia, egocentricidad y delirios).

9. Psicoticismo (relacionada con la homónima propuesta por Eysenck. Continuo que va desde la alienación Interpersonal leve - estilo de vida esquizoide - a los síntomas dramáticos de la psicosis).

El listado permite la obtención de tres índices globales que informan, en una puntuación única sobre el nivel de profundidad y dimensión de malestar psicológico: 
Índice de severidad global (GSI). Es un indicador del nivel actual de severidad del desorden. Combina el número de síntomas y la intensidad del estrés percibido.

Índice de malestar sintomatológico. Mide la intensidad de los síntomas e informa si el paciente los exagera o atenúa.

Índice de síntomas positivos. Considera la cantidad de síntomas informados. Es una medida de la cobertura de la sintomatologia, es decir, cuantas áreas afecta.

\section{Satisfacción con la vida}

Es una escala multítem ( 5 ítemes) con formato de respuesta likert en siete categorías y examina el grado de satisfacción global con la vida (Diener, Emmons, Larsen y Griffin, 1985). La escala es utilizada internacionalmente para la evaluación del bienestar en tanto componente cognitivo de la satisfacción. Diferentes estudios empíricos han demostrado su validez y fiabilidad (Castro Solano, 1999; Diener et al., 1985; Diener y Larsen, 1991; Larsen, Diener y Emmons, 1985). La mayoría de los estudios con este instrumento están basados en población adulta. Siguiendo las recomendaciones del autor, se utiliza la misma para población adolescente. Existen estudios locales con adolescentes que verificaron las propiedades psicométricas de la misma (Castro Solano, 2000). Se obtiene una puntuación media total que oscila entre 1 y 7 puntos, que indica el grado de satisfacción con la vida.

\section{Escala D-T}

Es una encuesta que evalúa el grado de satisfacción en cada una de las áreas vitales relevantes a la población objeto de estudio. Posee un ítem único para cada variable evaluada, con formato de respuesta likert en 7 opciones de respuesta. Está basada en la escala D-T (Delight - Terrible) de Andrews y Withey (1976). Existen estudios empíricos que utilizaron la misma con buenos resultados en población española (Castro Solano y Sánchez López, en prensa) y adolescente argentina (Castro 
Solano, 2000). Evalúa el grado de satisfacción alcanzado en cada una de las siguientes áreas:

- Estudios

- Familia

- Amigos

- Compañeros de estudio

- Salud Física

- Salud Psicológica

- Nivel de vida

Se obtiene una única puntuación por cada área que revela el grado de satisfacción alcanzado en cada aspecto de la vida considerado.

\section{Procedimiento}

A un grupo de estudiantes de la asignatura Técnicas Psicométricas de la Carrera de Psicología de la Universidad de Buenos Aires, durante el curso 1999 se les solicitó que administraran cinco protocolos al azar en población no consultante, que residiera en la ciudad de Buenos Aires y el Gran Buenos Aires. Los estudiantes habían recibido un entrenamiento previo en la administración de las pruebas. Dicha actividad era uno de los requisitos para aprobar la asignatura. Las actividades fueron coordinadas por uno de los autores (MMC). De la implementación de este procedimiento resultaron 524 protocolos que constituyeron el grupo de población general de la Región Buenos Aires incluido en este estudio. Varios protocolos fueron eliminados por contener respuestas incompletas o por no haber consignado datos sobre el sexo o la edad.

Los participantes de la Región Noroeste (Tucumán) fueron recogidos por un asistente de investigación, el cual recibió entrenamiento sobre la administración de las pruebas y su tabulación. La selección de los cursos fue intencional, dado que se trabajó con instituciones que aceptaron participar del proyecto. Los estudiantes trabajaron de forma anónima y voluntaria. 
En la Región Patagonia se trabajó de la misma manera, pero no fueron administradas las pruebas sobre satisfacción con la vida ni la escala D-T.

Los datos fueron analizados con el paquete estadístico SPSS, versión 8.01. Los cálculos de potencia estadística y tamaño del efecto fueron obtenidos mediante el programa Statistical Power Analysis (Cohen y Borenstein, 1988).

\section{Resultados}

\section{Estructura factorial de la escala BIEPS-J}

Para verificar empíricamente que los constructos se agrupaban según las dimensiones predichas teóricamente en los adolescentes estudiados, se llevó a cabo un análisis factorial de componentes principales de los 20 elementos de la BIEPS-J. Se comprobó previamente que la matriz de correlaciones era adecuada para este tipo de análisis (Test de esfericidad de Barttlet $=1343, p<.0001$; índice de adecuación muestral Káiser Meyer Olkin $=0.72$ ). El procedimiento sirvió para eliminar los elementos que saturaran muy alto en más de un factor, eliminándose y llevándose a cabo el proceso nuevamente. Siguiendo este procedimiento de forma iterativa se obtuvo la solución factorial que aquí se presenta, eliminándose siete elementos de los 20 iniciales. La solución demostró empíricamente que existían cuatro factores bien diferenciados que explicaban el $51 \%$ de la varianza y que eran relativamente independientes entre si según la predicción teórica en tanto componentes del bienestar psicológico de los adolescentes: control de situaciones, vínculos, proyectos y aceptación de si. El factor autonomía resultó poco claro, por lo tanto fue eliminado. Los coeficientes de confiabilidad son algo bajos, aunque se supone que este efecto tiene que ver no tanto con la homogeneidad de las dimensiones estudiadas sino con el bajo número de elementos por cada escala, ya que la confiabilidad total alcanza un valor bastante aceptable de 0.74. En el Cuadro 1 se presentan estos datos. 
Cuadro 1

Estructura Factorial de la BIEPS-J

\begin{tabular}{|c|c|c|c|c|}
\hline itemes. & $\begin{array}{l}\text { Factor } 1 \\
\text { Control }\end{array}$ & $\begin{array}{l}\text { Factor } 2 \\
\text { Vínculos }\end{array}$ & $\begin{array}{l}\text { Factor } 3 \\
\text { Proyectos }\end{array}$ & $\begin{array}{l}\text { Factor } 4 \\
\text { Aceptación }\end{array}$ \\
\hline $\begin{array}{l}\text { Confiabilidad } \\
\text { Alpha Total }=0.74\end{array}$ & 0.56 & 0.51 & 0.55 & 0.50 \\
\hline Item 20 & 0.73 & & & 0.13 \\
\hline Item 7 & 0.70 & & & 0.14 \\
\hline Item 17 & 0.57 & 0.10 & 0.14 & \\
\hline Item 2 & 0.53 & & & \\
\hline Item 13 & 0.13 & 0.63 & & \\
\hline Item 3 & & 0.79 & & \\
\hline Item 18 & & 0.59 & & 0.42 \\
\hline Item 19 & 0.21 & & 0.74 & \\
\hline Item 9 & & & 0.72 & \\
\hline Item 4 & & & 0.67 & 0.24 \\
\hline Item 15 & 0.20 & & & 0.72 \\
\hline Item 5 & & & 0.12 & 0.70 \\
\hline Item 10 & & 0.48 & & 0.51 \\
\hline
\end{tabular}

\section{Análisis correlacionales}

Para verificar que el instrumento evalúe bienestar psicológico y no otro constructo asociado se llevaron a cabo una serie de estudios correlacionales con otras pruebas que evalúan clásicamente bienestar y 
sintomatología clínica. Se había hipotetizado encontrar correlaciones positivas con otras mediciones de la satisfacción vital aunque no muy altas ya que el constructo presentado se considera distinto de las medidas clásicas de satisfacción. En la misma línea se esperaba encontrar correlaciones negativas con la presencia de síntomas psicopatológicos ya que las personas que autoperciben sintomatología psicológica experimentan en términos generales menor bienestar psicológico.

\section{BIEPS y Síntomas Clínicos}

Se correlacionaron la presencia de síntomas clínicos con el bienestar. En todos los casos se encontraron correlaciones negativas y estadísticamente significativas, excepto para dos escalas del SCL-90 (Los datos aparecen en el Cuadro 2). Las correlaciones negativas más altas se encontraron con las escalas de malestar psicológico y la severidad de sintomatología. Por lo tanto a mayor grado de psicopatología de los jóvenes había una disminución en la autopercepción del bienestar psicológico. La dimensión aceptación de sí es la que correlacionó más negativamente con la presencia de síntomas: aquellas personas que registraron mayor sintomatología se sentían más desilusionadas de sí mismas y estaban preocupadas acerca de sus cualidades personales, con deseos de ser diferentes a como eran. La dimensión Proyectos es la que menos se relaciona con la presencia de síntomas clínicos: la presencia de síntomas afectaba poco la percepción de propósitos y de significado de la vida o el tener proyectos personales.

\section{BIEPS y Satisfacción Vital}

Se calcularon las correlaciones entre la escala BIEPS y las puntuaciones del bienestar autopercibido evaluado a través de la escala de SWLS de E. Diener (1985) y con la satisfacción que experimentaban los adolescentes en cada área de su vida en particular mediante la escala D-T de Andrews y Withey (1976). Se había hipotetizado encontrar 


\section{Cuadro 2}

Correlación BIEPS y SCL-90-R

\begin{tabular}{|c|c|c|c|c|c|}
\hline$N=951$ & $\begin{array}{l}\text { BLPS-1 } \\
\text { Tolal }\end{array}$ & $\begin{array}{l}\text { Factor } 1 \\
\text { Control }\end{array}$ & $\begin{array}{l}\text { Factor? } \\
\text { Vinculos }\end{array}$ & $\begin{array}{l}\text { Factor } 3 \\
\text { Proyectos }\end{array}$ & $\begin{array}{l}\text { Factor } 4 \\
\text { aceptación }\end{array}$ \\
\hline Somatización & -0.30 & -0.18 & -0.21 & -0.12 & -0.27 \\
\hline Obsesivo- & -0.33 & -0.22 & -0.21 & -0.13 & -0.29 \\
\hline \multicolumn{6}{|l|}{ Compulsivo } \\
\hline Sensibilidad & -0.39 & -0.25 & -0.24 & -0.09 & -0.43 \\
\hline \multicolumn{6}{|l|}{ Interpersonal } \\
\hline Depresión & -0.39 & -0.21 & -0.27 & -0.14 & -0.40 \\
\hline Ansiedad & -0.35 & -0.21 & -0.26 & $-0.10^{\mathrm{a}}$ & -0.36 \\
\hline Hostilidad & -0.34 & -0.28 & -0.21 & -0.10 & -0.27 \\
\hline Fobia & -0.30 & -0.23 & -0.24 & $-0.05^{\mathrm{a}}$ & -0.27 \\
\hline Ideación Paranoide & -0.34 & -0.22 & -0.27 & -0.08 & -0.31 \\
\hline Psicoticismo & -0.37 & -0.25 & -0.30 & -0.09 & -0.33 \\
\hline Severidad Global & -0.40 & -0.27 & -0.29 & -0.11 & -0.37 \\
\hline Malestar Psicológico & -0.35 & -0.22 & -0.27 & -0.10 & -0.37 \\
\hline Síntomas Positivos & -0.32 & -0.23 & -0.26 & -0.11 & -0.25 \\
\hline
\end{tabular}

Nota. " La correlación no es significativa $p<.001$, como en los demás casos.

correlaciones positivas entre las puntuaciones de la BIEPS y las otras mediciones del bienestar aunque no muy altas, ya que la escala diseñada pretendía evaluar una dimensión diferente del bienestar psicológico. El resultado del análisis demuestra que en la medida que los jóvenes experimentan mayor bienestar psicológico se sienten mas satisfechos con la vida en general y con la satisfacción experimentada en cada una de las áreas vitales. Si bien se obtuvieron correlaciones positivas, éstas no resultaron muy elevadas acorde a lo esperado (entre 0.20 y 0.30 ). Respecto de cada factor en particular, los más correlacionados con las mediciones tradicionales del bienestar fueron vínculos y aceptación de sí mismo. El factor control prácticamente no estaba relacionado con la satisfacción 
tal como resultaba con las pruebas clásicas. Es decir, que en términos genéricos, la dimensión relacionada con las habilidades para manejar el entorno y satisfacer las propias necesidades según los propios valores no estaban capturadas por las pruebas de satisfacción clásicas. Los datos completos se presentan en el Cuadro 3.

En segundo término se llevó a cabo un análisis factorial de componentes principales. El propósito de este análisis fue verificar la existencia de dimensiones subyacentes diferentes que explicasen la variación de las puntuaciones del bienestar psicológico. Para verificar que la prueba medía un constructo diferente se tenía que encontrar altos pesos factoriales de la BIEPS en un factor independiente respecto de las otras pruebas que medían bienestar. Se llevó a cabo un análisis de componentes principales y se extrajeron 2 factores según el método Káiser, que en conjunto explicaban el $45 \%$ de la varianza. En el primer factor tenían altos pesos factoriales la escala SWLS y la satisfacción por áreas, y en el segundo factor, las puntuaciones totales y por escala de la BIEPS, confirmándose la hipótesis preliminar. En el Cuadro 4 aparecen los datos.

Cuadro 3

Correlaciones BIEPS y Satisfacción Vital

\begin{tabular}{|c|c|c|c|c|c|}
\hline$N=951$ & $\begin{array}{l}\text { BIEPS-J } \\
\text { Total }\end{array}$ & $\begin{array}{l}\text { Factor } 1 \\
\text { Control }\end{array}$ & $\begin{array}{l}\text { Factor } 2 \\
\text { Vinculos }\end{array}$ & $\begin{array}{l}\text { Factor } 3 \\
\text { Proyectos }\end{array}$ & $\begin{array}{l}\text { Factor } 4 \\
\text { Aceptación }\end{array}$ \\
\hline SWLS (Diener) & $0.31 * *$ & 0.07 & $0.22^{* *}$ & $0.18 * *$ & $0.31 * *$ \\
\hline Sat. Estudios & $0.13 * *$ & 0.06 & 0.07 & $0.16^{* *}$ & 0.02 \\
\hline Sat. Familia & $0.19 * *$ & 0.06 & $0.22 * *$ & $0.11 * *$ & 0.09 \\
\hline Sat. Amigos & $0.22 * *$ & 0.03 & $0.44^{* *}$ & 0.01 & $0.13 * *$ \\
\hline Sat. Compañeros & $0.17 * *$ & 0.03 & $0.28 * *$ & 0.04 & 0.29 \\
\hline Sat. Salud Física & $0.27^{* *}$ & $0.12 * *$ & $0.14 * *$ & $0.14^{* *}$ & $0.28 * *$ \\
\hline Sat. Psicológica & $0.26 * *$ & 0.10 & $0.14 * *$ & $0.28 * *$ & $0.23 * *$ \\
\hline Sat. Nivel de Vida & $0.26^{* * *}$ & 0.05 & $0.24 * *$ & $0.17 * *$ & $0.21 * *$ \\
\hline
\end{tabular}

${ }^{* *} p<.01 * p<.05$ 
Cuadro 4

Análisis Factorial BIEPS y Satisfacción Vital

\begin{tabular}{|lll|}
\hline \multicolumn{1}{|c|}{ Escalas } & Factor 1 & Factor 2 \\
\hline Sat. Nivel de Vida & $\mathbf{0 . 7 4}$ & 0.12 \\
SWLS & $\mathbf{0 . 7 2}$ & 0.20 \\
Sat. Familia & $\mathbf{0 . 6 9}$ & \\
Sat. Salud & $\mathbf{0 . 5 9}$ & 0.18 \\
Psicologica & & \\
Sat. Salud Fisica & $\mathbf{0 . 5 5}$ & 0.22 \\
Sat. Amigos & $\mathbf{0 . 5 5}$ & 0.13 \\
Sat. Estudios & $\mathbf{0 . 5 0}$ & \\
Sat. Compañeros & $\mathbf{0 . 4 7}$ & \\
BIEPS-J Total & 0.18 & $\mathbf{0 . 9 8}$ \\
Factor 1 Control & & $\mathbf{0 . 7 1}$ \\
Factor 2 & 0.18 & $\mathbf{0 . 6 2}$ \\
Aceptacion & & \\
Factor 3 - Vinculos & 0.30 & $\mathbf{0 . 5 7}$ \\
Factor 4 -Proyectos & & $\mathbf{0 . 5 6}$ \\
\hline
\end{tabular}

\section{Perfiles diferenciales de bienestar según sexo, edad y contexto}

Para analizar si existían diferencias por edad, por sexo y contexto en cada una de las puntuaciones en los factores del bienestar, se llevó a cabo un análisis multivariado de la varianza (MANOVA) con un diseño 2 (sexo $=$ varón o mujer) $\times 6$ (edades $=13$ a 18) $\times 3$ (lugares de residencia $=$ Buenos Aires, Noroeste y Patagonia).

En cuanto a la influencia del sexo, el análisis realizado arrojó diferencias estadísticamente significativas entre el vector de medias am- 
bos grupos $\left(\lambda\right.$ de Wilks $\left.=0.95, F(4,1194)=5.91, p<.001, \eta^{2}=0.05\right)$. Por lo tanto el contraste de las medias arroja diferencias en la mayoría de las escalas de la BIEPS. Siguiendo las indicaciones de Cohen (1991) para la interpretación del tamaño del efecto del contraste multivariado implementado se puede considerar la magnitud de estas diferencias como muy pequeña ( $\eta^{2}$ alrededor de 0.10 ). Se atiende al porcentaje de varianza explicada por la diferencia de sexos $\left(r^{2}\right)$, se aprecia que no alcanzó valores superiores al $3 \%$ o $4 \%$ de la variabilidad total. El resto de la varianza que no estaba explicada por esta diferencia estaría en función de otros factores distintos, limitando por lo tanto la significación práctica de las diferencias observadas.

El análisis no arrojó diferencias estadísticamente significativas entre los vectores de medias de los diferentes grupos de edades, tomando en cuenta los resultados de forma agregada ( $\lambda$ de Wilks $=0.96, F(20$, $3961)=1.48, p>.05, \eta^{2}=0.01$ ). Por lo tanto puede afirmarse que el bienestar psicológico, tomando en cuenta las dimensiones estudiadas, se mantuvo estable en los grupos estudiados y éstos no estuvieron afectados por esta variable en el rango de edad considerado.

En cuanto a la influencia del contexto, el análisis arrojó diferencias estadísticamente significativas entre los vectores de medias de las escalas de la BIEPS para los diferentes contextos $(\lambda$ de Wilks $=0.95, F(8,2388)$ $=6.92, p<.001, \eta^{2}=0.02$ ), aunque el porcentaje de varianza explicada fue muy pequeña y el tamaño del efecto de las diferencias también lo es (alrededor del 2\%).

No se observó interacción entre el sexo y la edad $(\lambda$ de Wilks $=$ $\left.0.98, F(20,3961)=0.95, p>.05, \eta^{2}=0.004\right)$; el sexo y el lugar de residencia $\left(\lambda\right.$ de Wilks $\left.=0.99, F(8,2388)=0.82, p>.05, \eta^{2}=0.003\right)$; la edad y el lugar de residencia $(\lambda$ de Wilks $=0.95, F(40,4529)=1.28, p>$ $\left..05, \eta^{2}=0.011\right)$ y el sexo, la edad y la residencia en conjunto $(\lambda$ de Wilks $\left.=0.95, F(40,4529)=1.24, p>.05, \eta^{2}=0.010\right)$. Por lo tanto no existen diferencias en la percepción del bienestar psicológico entre los sexos, los diferentes grupos de edades y los contextos. 


\section{Perfiles diferenciales de Bienestar de Adolescentes en riesgo}

Para verificar si el bienestar psicológico en tanto capacidad de mantener buenos vínculos, control sobre el entorno, tener proyectos y autoaceptación de sí era considerado un factor protector de la salud mental se tenía que encontrar diferencias en estas dimensiones entre aquellos jóvenes que no registraron trastornos psicológicos y aquellos que se encontraron en situación de riesgo psicológico. Se trabajó sólo con los jóvenes residentes en Buenos Aires y Tucumán. Para ello se dividieron a los jóvenes en dos grupos bien diferenciados: aquellos que registraban alta sintomatología y aquellos que no registraban síntomas. Para poder agrupar a los jóvenes previamente se llevó a cabo un análisis de clusters de perfiles. La mejor solución encontrada era la de cuatro agrupaciones. El primer grupo integrado por la mayoría de los participantes $(n=427)$, la mayoría de los participantes obtuvo bajas puntuaciones en todas las escalas. En el segundo grupo $(n=139)$, la mayoría de los participantes obtuvo puntuaciones a más de una desviación estándar en todas las escalas. El tercer grupo $(n=347)$ obtuvo puntuaciones promedio y en el cuarto grupo $(n=39)$, la mayoría de los participantes obtuvo puntuaciones muy altas a más de dos desviaciones. El grupo 1 se consideró que no tenía trastornos psicológicos dado la ausencia de síntomas clínicos. Los grupos 2 y 4 registraron síntomas de moderados a graves. Por lo tanto quedaron dos grandes grupos de jóvenes: los que registraron sintomatología clínica (grupos 2 y 4) y los que no registraron trastornos psicológicos (grupo 1).

Para verificar si existían diferencias en cada grupo respecto de las puntuaciones en la BIEPS se llevó a cabo un análisis multivariado de la varianza (MANOVA). Se ingresó como variables dependientes las puntuaciones de la BIEPS y como factores la pertenencia a cada uno de los grupos (con trastorno/sin trastorno).

El análisis realizado arrojó diferencias estadísticamente significativas entre el vector de medias ambos grupos $(\lambda$ de Wilks $=0.74, F(4,574)=$ $57.17, p<.001, \eta^{2}=0.26$ ). Por lo tanto el contraste de las medias arroja diferencias en la mayoría de las escalas de la BIEPS. Siguiendo las 
indicaciones de Cohen (1991) para la interpretación del tamaño del efecto del contraste multivariado implementado podemos considerar la magnitud de estas diferencias como amplia $\left(\eta^{2}\right.$ alrededor $\left.>0.25\right)$. Si se atiende al porcentaje de varianza explicada se puede apreciar que ronda los valores del $22 \%$ de la variabilidad total.

Para analizar qué escalas de la BIEPS discriminaban mejor entre los jóvenes que tenían más y menos trastornos psicológicos, se llevó a cabo un análisis discriminante, según las recomendaciones de Tatsuoka (1971). Se obtuvo una función discriminante que era significativa ( $\lambda$ de Wilks $=$ $0.74, x^{2}=179.38, g l=4, p<.001$ ) y explicaba un $35 \%$ de la varianza. Los resultados del análisis discriminante aparecen en el Cuadro 5. Las variables que más diferencian a los grupos fueron Aceptación de sí ( $\mathrm{r}$ con la función $=0.82$ ) y Vínculos ( $\mathrm{r}$ con la función $=0.61$ ). Es decir que los jóvenes que registraron sintomatología clínica grave y moderada obtuvieron menor bienestar psicológico en relación con la apreciación general de sí mismos y con la calidad de los vínculos que establecieron. Sentían que su vida no valía nada, mostraron inseguridad acerca de sus cualidades personales, les costaba establecer buenos vínculos con los demás y se aislaban de sus pares.

\section{Cuadro 5}

Análisis Discriminante Jóvenes con y sin Trastornos Psicológicos

\begin{tabular}{|lc|}
\hline BIEPS & $\begin{array}{c}\text { Correlación con la función } \\
\text { discriminante }\end{array}$ \\
\hline BIEPS Total & 0.87 \\
Aceptación & 0.82 \\
Vínculos & 0.61 \\
Control & 0.53 \\
Proyectos & 0.24 \\
\hline
\end{tabular}




\section{Discusión}

Se cuenta con una técnica autoadministrable válida y confiable para la evaluación de la autopercepción del Bienestar Psicológico. Los datos presentados y analizados sustentan que sólo se debe utilizar la puntuación total de la escala BIEPS cuya confiabilidad es aceptable (0.74).

Cuando el evaluador analice esta puntuación global, se recomienda el análisis cualitativo de las respuestas dadas a los ítemes agrupados según cada una de las cuatro categorías: control de la situación, vínculos, proyectos y aceptación de sí mismo. Este puntaje global directo o bruto se interpreta en función de transformaciones percentilares, sin tener en cuenta diferencias según género o edad.

Los datos analizados indican la correlación negativa entre el Bienestar Psicológico y la presencia de síntomas psicopatológicos. La presencia de psicopatologías afecta especialmente las dimensiones Aceptación de Uno Mismo y Tipos de Vínculos Psicosociales que se pueden concretar. Los adolescentes con problemas emocionales severos no se reconocen sintiéndose psicológicamente bien, a mayor presencia de psicopatologías, menor autopercepción de bienestar psicológico. La aceptación de sí mismo revela una correlación negativa con la presencia de síntomas, presencia de sentimientos de desilusión y preocupaciones por ser diferente a los demás. Es importante destacar que la existencia de síntomas psicológicos parece afectar poco el poder tener propósitos vitales y proyectos personales.

Las escalas clásicas sobre Satisfacción con la Vida y Satisfacción con las Áreas Vitales resultan indicadores poco válidos para una evaluación del bienestar psicológico autopercibido; existen dimensiones subyacentes diferentes en las técnicas empleadas.

Las variables género, edad y contexto sociocultural no parecen afectar la percepción subjetiva del bienestar psicológico en la adolescencia. 
Evaluación del bienestar psicologico en estudiantes adolescentes argentinos

\section{Referencias}

Adams, R. (1992). Is happiness a home in the suburbs?: The influence of Urban versus Suburban Neighborhoods on psychological health. Journal of Community Psychology, 20, 353-372.

Andrews., F. Withey, S. (1976). Social indicators of well being: American's perceptions of life quality. Nueva York: Plenum.

Antonovsky, A. (1988). Unraveling the mystery of health. San Francisco: Jossey-Bass.

Argyle, M. (1987). The psychology of happiness. Londres: Methuen.

Avia, M. y Vazquez, C. (1998). Optimismo Inteligente. Madrid: Alianza Editorial.

Beiser, M. y Collomb, H. (1981). Mastering change: epidemiological and case studies of Senegal. American Journal of Psychiatry, 138, 455-459.

Campbel, A., Converse, P. y Rodgers, E. (1976). The quality of american life: percepcions, evaluations and satisfactions. Nueva York: Russel Sage Foundation.

Castro Solano, A. (1999). Diferencias individuales en la autopercepción del bienestar, los objetivos de vida y los estilos de personalidad en estudiantes universitarios españoles. Memoria de Licenciatura, Universidad Complutense, Madrid.

Castro Solano, A. (2000). Estilos de personalidad, objetivos de vida y satisfacción vital. Un estudio comparativo con adolescentes argentinos. Tesis doctoral, Universidad Complutense, Madrid.

Castro Solano, A. y Sánchez López, M. (en prensa). Objetivos de Vida y Satisfacción. Psicothema.

Cohen, J. (1991). Statistical power analysis for the behavioral sciences. Nueva Jersey: Erlbaum.

Cohen, J. y Borenstein, M. (1988). Statistical power analysis: A computer program. Nueva Jersey: Erlbaum.

Costa, P. y McCrae, R. (1980). Influence of extraversion and neuroticism on subjective well being: happy and unhappy people. Journal of Personality and Social Psychology, 38 (4), 668-678. 
Costa, P. y McCrae, R. (1984). Personality as a life long determinant of well-being. En C. Malatesta y C. Izard (Eds.), Affective Procceses in adult development and aging. (pp. 141-156). Beverly Hills: Sage.

Costa, P., McCrae, R. y Zonderman, A. (1987). Environmental and dispositional influences on well-being: Longitudinal follow-up of an American national sample. British Journal of Psychology, 78, 299306.

Crider, D., Willits, F. y Kanagy, C. (1991). Rurality and Well-being during the middle years of life. Social Indicators Research, 24, 253268.

Cummins, R. (1998). The second approximation to an international standard for life satisfaction. Social Indicators Research, 43, 307-334.

Cheng, A. (1989). Urbanization and minor psychiatric morbidity: A community study in Taiwan. Social Psychiatry and Psychiatric Epidemiology, 24, 309-316.

Diener, E. (1994). Assessing subjective well-being: Progress and opportunities. Social Indicators Research, 31, 103-157.

Diener, E. y Diener, M. (1995). Cross-cultural correlates of life satisfaction and self-esteem. Journal of Personality and Social Psychology, $68(4), 653-663$.

Diener, E., Emmons, R., Larsen, R. y Griffin, S. (1985). The satisfaction with Life Scale. Journal of Personality Asessment, 49 (1), 71-75

Diener, E. y Larsen, R. (1991). The experience of emotional well-being. En M. Lewis y J.M. Haviland (Eds.), Handbook of emotions (pp. 405-415) (Capítulo 28). Nueva York: Guilford.

Diener, E., Suh, E. y Oishi, S. (1997). Recent findings on subjective well-being. Indian Journal of Clinical Psychology (publicación on-line).

Dohrenwend, B. P. y Dohrenwen, B. S. (1974). Psychiatric disorders in urban setttings. En G. Caplan (Ed.), American handbook of psychiatry (pp. 424-427). Nueva York: Basic Books.

Emmons, R., Diener, E. (1985). Personality correlates of subjective well being. Personality and Social Psychology Bulletin, 11 (1), 89-97.

Fischer, C. (1973). On urban alienation and anomie: Powerlessnes and social isolation, American Sociological Review, 28, 311-326. 
Frenz, A., Carey, M., Jorgensen, R. (1993). Psychometric evaluation of Antonovsky's sense of coherence scale. Psychological Assessment, $5(2), 145-153$.

Harris, C. (1984). Mental illness in inner London. British Medical Journal, 288, 1425-1426.

Headey, B. y Wearing, A. (1989). Personality, life events and subjective well being, Toward a dynamic equilibrium model. Journal of Personality and Social Psychology, 57 (4), 731-739.

Hotard, S., Mc Feather, R., Mc Whirter, R. y Stegall, M. (1989). Interactive effects of extraversion, neuroticism and social relationships on subjective well-being. Journal of Personality and Social Psychology, 57, 321-331.

Hynson, L. (1975). Rural-urban differences in satisfaction among the elderly. Rural Sociology, 40, 64-66.

Kushman, J. y Lane, S. (1980). A multivariate analysis of factors affecting perceived life satisfaction and psychological well being among the elderly. Social Sciences Quaterly, 61, 264-277.

Larsen, R., Diener, E. y Emmons, R. (1985). An evaluation of subjective well-being measures. Social Indicators Research, 17, 1-17.

Larsen, R. y Ketelaar, T. (1991). Personality and suceptibility to positive and negative emotional states. Journal of Personality and Social Psychology, 61, 132-140.

Lucas, R. E., Diener, E. y Suh, E. (1996). Discriminant validity of wellbeing measures. Journal of Personality and Social Psychology, 71, 616-628.

Lykken, D. y Tellegen, A. (1996). Happiness is a stochastic phenomenon. Psychological Science, 7, 186-189.

Milgram, S. (1970). The experience of living in cities. Science, 167, 14611468.

Mueller, D. (1981). The current status of urban-rural differences in psychiatric disroder: An emerging trend for depresssion. Joumal of Nervous and Mental Disease, 169, 18-27.

Neff, J. (1983). Urbanicity and depression reconsidered: The evidence regarding depressive symptomatology. Journal of Nervouse and Mental Disease, 171, 546-552. 
Pagliccia, N., Apland, L. y Kazanjian, A. (1995). The impact of professional and personal satisfaction on perceptions of rural and urban: Some analytic evidence. Social Indicators Research, 34, 367-376. Pychyl, T. y Little, B., (1998). Dimensional specificity in the prediction of subjective well-being: personal projects in the pursuit of the Phd. Social Indicator Research, 45, 423-473.

Reker, G., Peacock, E. y Wong, P. (1987). Meaning and purpose in life and well-being: a life span perspective. Journal of Gerontology, $42(1), 44-49$.

Ryff, C. (1989). Happiness is everything, or is it? Exploration son the meanin of psychological well-being. Journal of Personality and Social Psychology, 57 (6), 1069-1081.

Ryff, C. y Keyes, C. (1995). The structure of psychological well-being revisited. Journal of Personality and Social Psychology, 69 (4), 719-727.

Sarason, S. B. (1974). The psychologicall sense of community. San Francisco: Josey Bass.

Sauer, W., Shenan, J. y Boymel, C. (1976). Rural-urban differences in satisfaction among the elderly: a reconsideration. Rural Sociology, 41, 269-275.

Schumutte, P. y Ryff, C. (1997). Personality and well being: Reexamining methods and meanings. Journal of Personality and Social Psychology, 73 (3), 549-559.

Srole, L. (1972). Urbanization and Mental Health: Some reformulations. American Scientist, 60, 576-583.

Strack, F. y Argyle, M. y Schwartz, N. (Eds.) (1991). Subjective wellbeing. Oxford: Pergamon Press.

Taylor, S. y Brown, J. (1988). Illusion and well being: A social psychological perspective on mental health. Psychological Bulletin, 103 (2), 193-210.

Tellegen, A., Lykken, D., Bouchard, T. J., Wilcox, K. J., Segal, N. J. y Rich, S. (1988). Personality similarity in twins reared apart and together. Journal of Personality and Social Psychology, 54, 10311039. 
Evaluación del bienestar psicológico en estudiantes adolescentes argentinos

Triandis, H. (1989). The self and social behavior in differing cultural contexts. Psychological Review, 96 (3), 506-520.

Triandis, H. (1994). Culture and social behavior. Nueva York: Mc GrawHill.

Veenhoven, R. (1988). The utility of happiness. Social Indicators Research, 20, 333-354.

Veenhoven, R. (1991). Is happiness relative? Social Indicators Research, 24, 1-34.

Veenhoven, R. (1993). Is happiness a trait? Test of the theory that a better society does not make people any happier. Social Indicators Research, 32, 101-160.

Veenhoven, R. (1995). Developments in Satisfaction Research, Social Indicators Research, 37, 1-46. 


\section{ANEXO}

\section{Escala BIEPS}

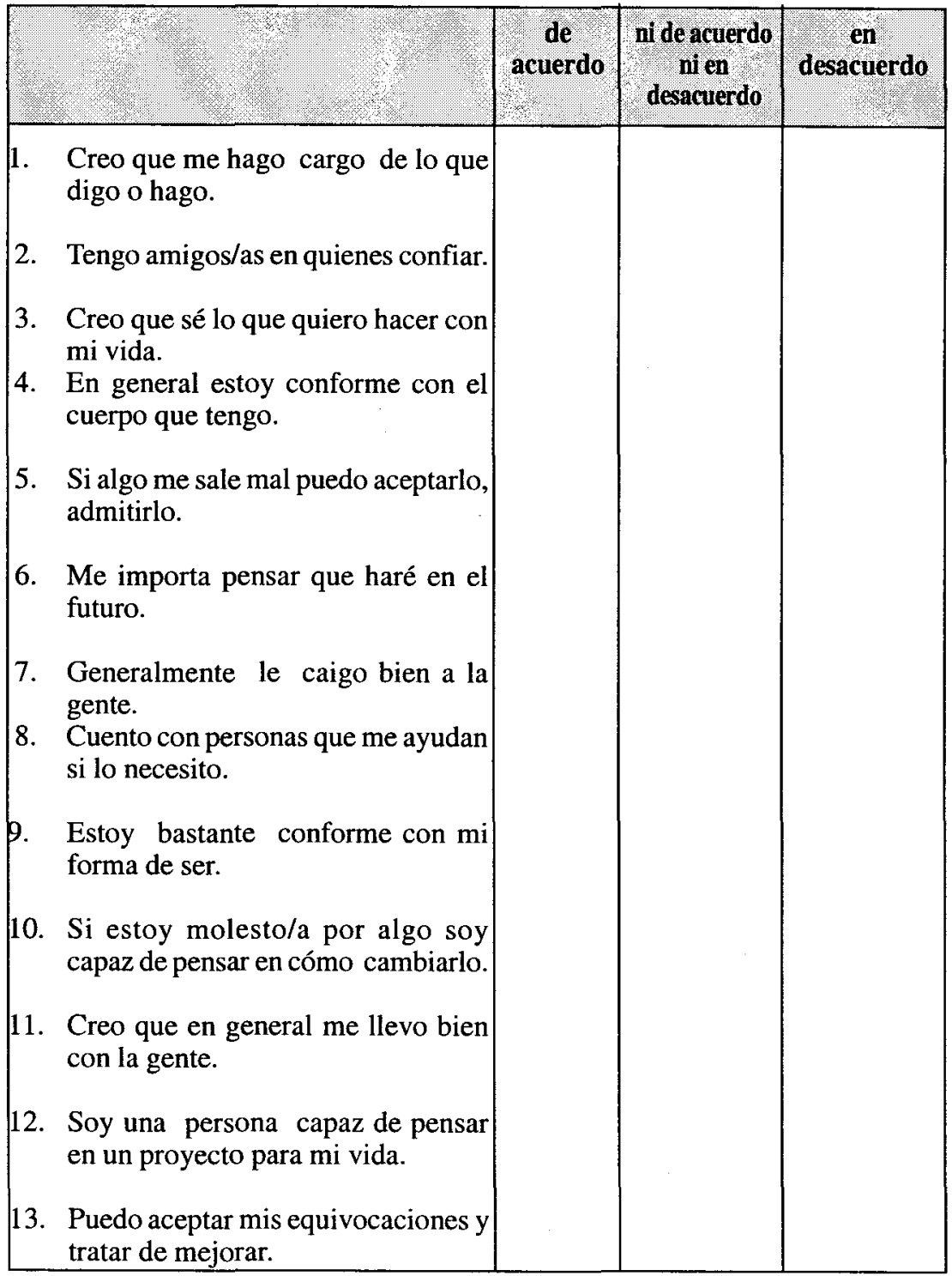

